\title{
(3) Plasma ADH Levels in Congestive Heart Failure
}

\author{
Yoko Yamane, M.D. and Toshiya Shiota, M.D. \\ The Second Department of Internal Medicine, \\ Kansai Medical School
}

The role of $\mathrm{ADH}$ in the failure of excretion of water and consequent edema formation accompanying congestive heart failure has been the subject of considerable recent investigations. As reported previously ${ }^{1)}$, plasma ADH increased in $45 \%$ of cases with right-sided heart failure and there was a statistically significant correlation between $\mathrm{ADH}$ levels and right ventricular enddiastolic pressure or right atrial pressure. The present study was undertaken to elucidate the effect of elevated venous pressure on plasma $\mathrm{ADH}$ level and the role of $\mathrm{ADH}$ in retention of water in congestive heart failure.

\section{Methods}

I. Clinical observations

Changes in plasma ADH levels after treatment with digitalis or furosemide ( $40 \mathrm{mg}$, orally) were studied in patients with heart failure. ADH levels were measured after fluid deprivation for 13 hours in cases with mitral stenosis and other heart diseases.

II. Animal experiments

1) Acute and chronic caval vein constriction

A balloon catheter was inserted into the superior and inferior caval vein under anesthesia. The balloon was inflated for 10 minutes and at the end of this period blood was taken from the superior caval vein. Pressure of right and left heart was recorded.

In chronic experiments a teflon tape was placed around the thoracic inferior caval vein according to McKee's report $t^{2)}$ in 11 dogs. In 8 out of 11 dogs, blood was taken from a peripheral vein under anesthesia and in 3 from the superior caval vein without anesthesia.

2) Experimental tricuspid insufficiency and pulmonary artery stenosis

One to two cusps of the tricuspid valve were removed with bioptom in $3 \mathrm{dogs}$ and $\mathrm{ADH}$ was measured 10 to 15 minutes and about 2 hours after operation. Pressure of right and left heart was recorded.

In one dog, as attempts to make tricuspid insufficiency with bioptom were unsuccessful, the chest was opened and a teflon tape was placed around the main pulmonary artery. In 2 other dogs a small incision was made on the right ventricular 
wall and the chordae which support the tricuspid cusps were cut. Sixteen to 44 days after operation blood was taken and cardiac catheterization and cineangiocardiography were performed.

Plasma ADH, plasma osmolality, plasma electrolytes, hematocrit and circulating blood volume were measured simultaneously. ADH was extracted from plasma according Yoshida's method ${ }^{32}$ and bioassay was carried out using experimental diabetes insipidus rats $^{4}$. In animal experiments mongrel dogs weighing 9.5 to $30 \mathrm{~kg}$ were used.

\section{Results and Discussion}

$\mathrm{ADH}$ normalized after digitalization or furosemide administration as Fig. 1 shows in cases whose venous pressure and circulating blood volume had been increased. When circulating blood volume did not change in spite of increased urine excretion, there would occur shift of body fluid from the extravascular to intravascular space, andithus $\mathrm{ADH}$ level did not change.
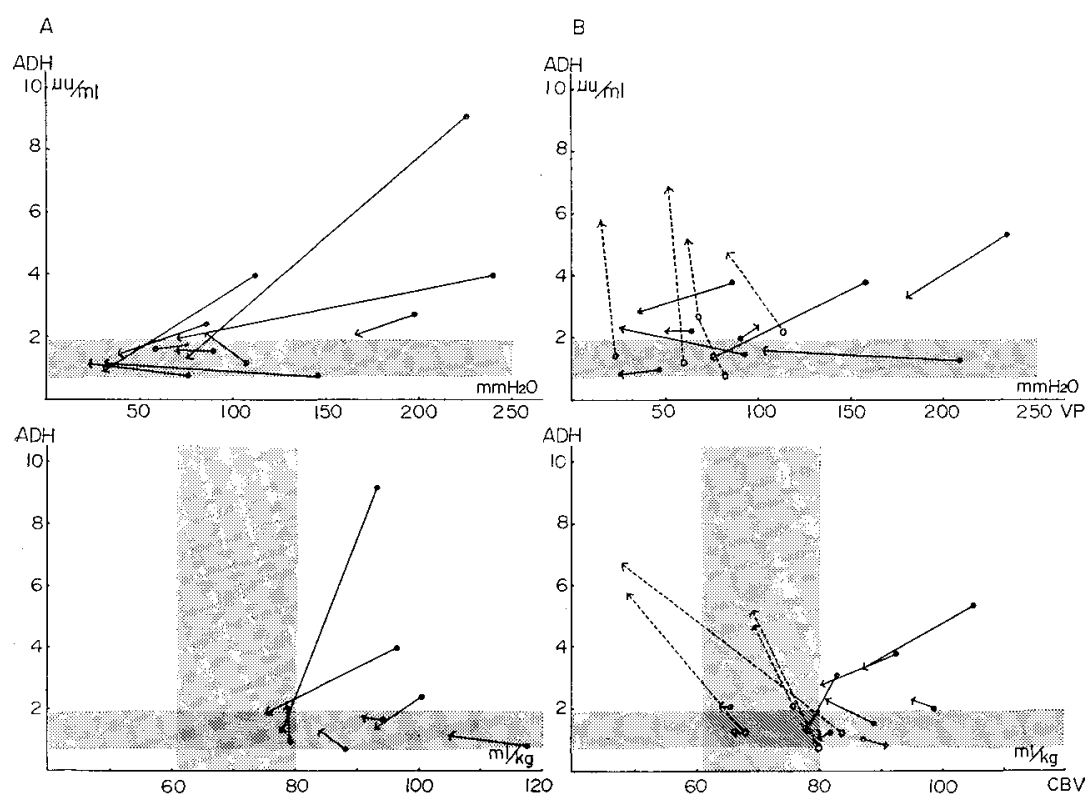

Fig. 1A. Effect of digitalis on plasma ADH level in patients with congestive heart failure.

1B. Effect of oral administration of furosemide at the dosage of $40 \mathrm{mg}$ on plasma $\mathrm{ADH}$ level.

The shaded area means the normal range.

$\mathrm{VP}$ : venous pressure, $\mathrm{CBV}$ : circulating blood volume,

$\cdots \rightarrow$ : normal controls, $\longrightarrow$ : patients with congestive heart failure. 
In cases with pure mitral stenosis plasma ADH levels were significantly lower than in cases with mitral insufficiency or other heart diseases regardless of presence or absence of auricular fibrillation.

II. Animal experiments

1) Acute and chronic caval vein constriction

Plasma ADH increased slightly to moderately without significant changes in the lef $t$ ventricular pressure and mean aortic pressure and it increased remarkably when the left ventricular pressure lowered significantly with balloon inflation. This suggests that in the former left atrial stretch receptor exerts an important influence on the level of $\mathrm{ADH}$ and in the latter arterial baroreceptor should be stimulated during decrease in venous return. There was significant correlation between plasma. $\mathrm{ADH}$ level and distal venous pressure in only one dog.

In 9 out of 11 dogs with chronic thoracic inferior caval vein constriction, ascites and edema in the lower part of the body occurred. Plasma ADH levels were within normal range in anesthetized dogs and in 2 out of 3 unanesthetized dogs ADH increased significantly as Fig. 2 shows. In one dog hyponatremia occurred but it was uncertain whether the cause of hyponatremia is due to the release of ADH or not. In one unanesthetized dog plasma $\mathrm{ADH}$ increased remarkably and decrease in circulating blood volume was suspected.
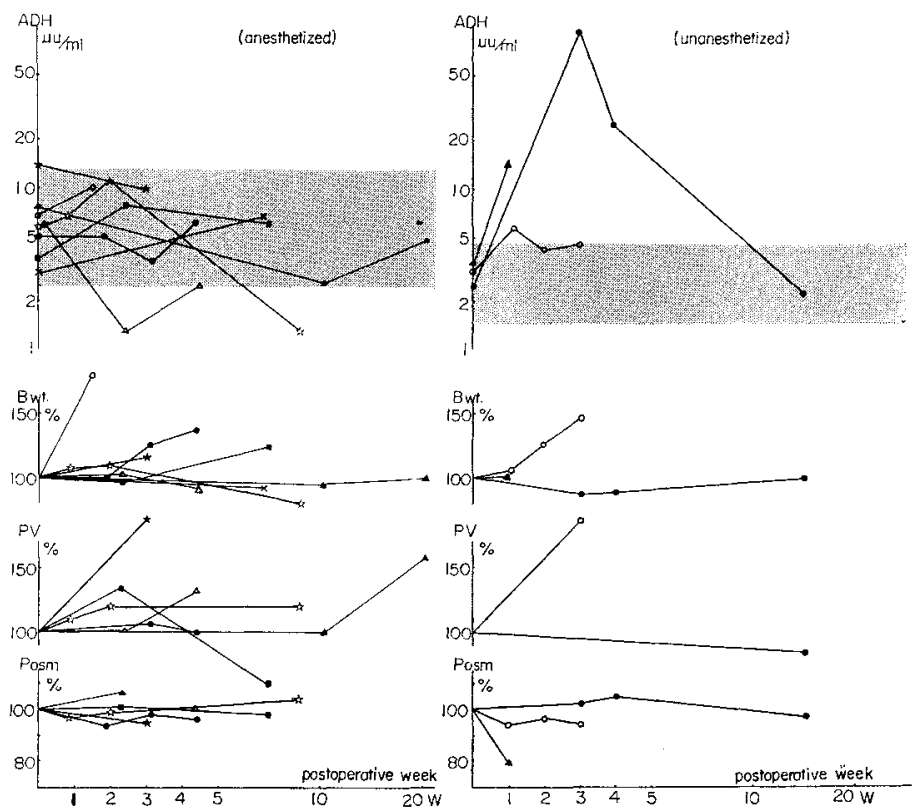

Fig. 2. Effect of chronic thoracic caval vein constriction on plasma $A D H$ level in dogs with and without anesthesia.

The shaded area means the normal range.

Bwt. : body weight, PV: plasma volume, Posm : plasma osmolality, W: weeks. 
There was no correlation between ADH levels and the degree of ascites.

2) Experimental tricuspid insufficiency and pulmonary artery stenosis

Plasma ADH increased in acute tricuspid insufficiency as Fig. $3 \mathrm{~A}$ shows. At the same time left atrial pressure and/or left ventricular enddiastolic pressure decreased slightly. In dogs with pulmonary artery stenosis or tricuspid insufficiency, ADH levels were within normal range although right ventricular enddiastolic pressure and right atrial pressure elevated (Fig. 3B).

Even at the compensated stage it is known that the kidney overabsorbs salt and water and the redistribution of the renal blood flow occurs. The exact mechanism of increased $\mathrm{ADH}$ in right heart failure is uncertain. When the right heart fails, the amount of body fluid increases, generalized systemic venous congestion occurs, the body fluid shifts from the intravascular to extravascular space and edema
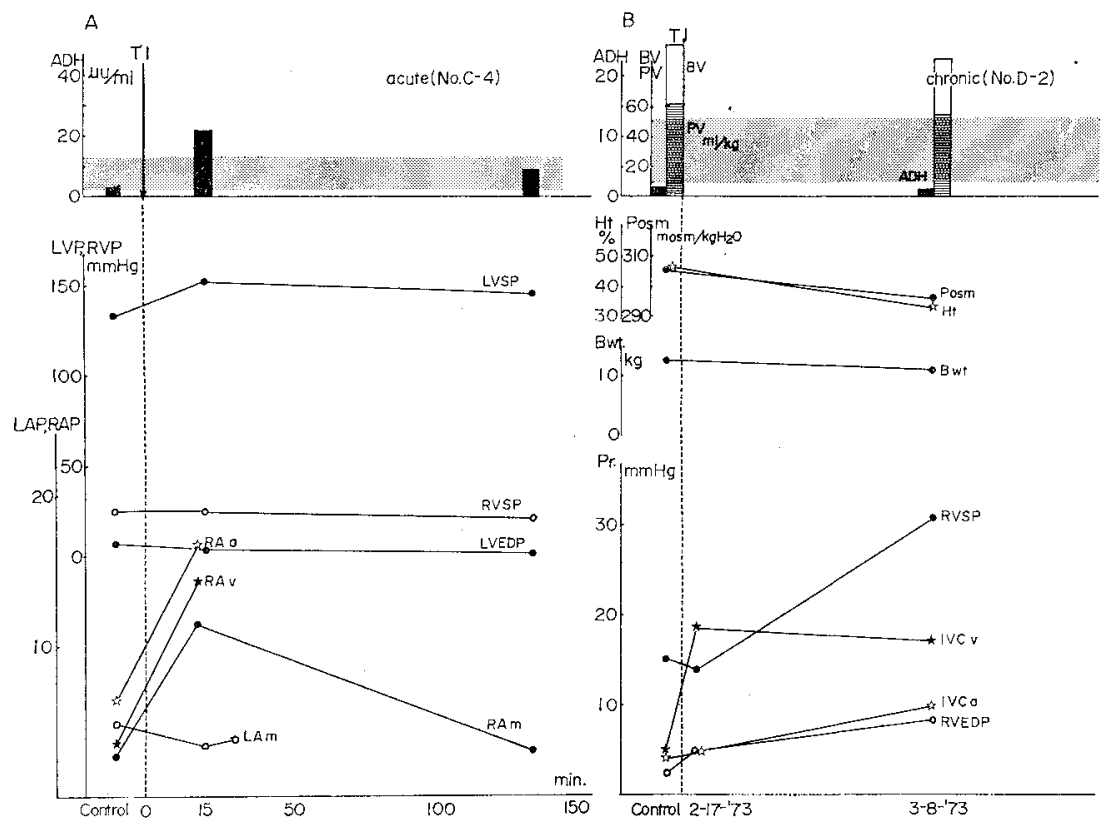

Fig. 3A. Change in plasma ADH level and pressure of right and left heart during acute tricuspid insufficiency with bioptom in a dog.

3B. Plasma $\mathrm{ADH}$ level and pressure of the right heart before and 19 days after making experimental tricuspid insufficiency. The shaded area means the normal range.

LVP : left ventricular pressure, RVP : right ventricular pressure, LVSP : left ventricular systolic pressure, RVSP : right ventricular systolic pressure, LVEDP : left ventricular enddiastolic pressure, LAP : left atrial pressure, RAP : right atrial pressure, RAa: "a" wave of right atrial pressure, RAv: "v" wave of right atrial pressure, RAm: right atrial mean pressure, LAm: left atrial mean pressure, TI : tricuspid insufficiency, BV: blood volume, PV : plasma volume, Ht : hematocrit, Posm: plasma osmolality, Bwt : body weight, Pr: pressure, IVCV: "v" wave of inferior caval vein pressure, IVCa: "a" wave of inferior caval vein pressure, RVEDP : right ventricular enddiastolic pressure, min.: minutes. 
accumulates. An elevation of venous pressure leads to an increase in extravasation of body fluid and a decrease in venous return with increased oncotic pressure. These changes in body fluid stimulate the release of ADH and further renal retention of water, which leads to hypervolemia and elevation of venous pressure, thus constituting a vicious circle. When this vicious circle is stopped by digitalis or diuretics, elevated $\mathrm{ADH}$ will decrease.

\section{Conclusion}

From the data above-mentioned and previously reported ${ }^{1>} \mathrm{ADH}$ seems not to be a primary factor in retention of water in congestive heart failure. It appears that ADH increases perhaps in response to "deficient" circulating blood volume and cardiac output.

\section{References}

1) Yamane, Y.: Plasma ADH level in patients with chronic congestive heart failure. Jap. Circul. J. 32: 745, 1968.

2) McKee, F. W., Schilling, J.A., Tishkoff, G.H. and Hyatt, R. E. : Experimental ascites. Effect of sodium chloride and protein intake on protein metabolism of dogs with constricted inferior vena cava. Surg. Gynec. Obst. $89: 529,1949$.

3) Yoshida, S., Motohashi, K., Ibayashi, H. and Okinaka, S.: Method for the assay of antidiuretic hormone in plasma with a note on the antidiuretic titer of human plasma. J. Lab. Clin. Med. 62: 279, 1963.

4) Yamane, Y. and Kunishige, K. : Bioassay of ADH on the experimental diabetes insipidus rats. Jap. Circul. J. $30: 1381,1966$. 